\title{
Psicosis del personal de salud en tiempos de COVID-19
}

Psychosis of health care personnel in times of COVID-19

Psicose do pessoal de saúde no tempo de COVID-19

Jesús Salvador Sánchez Díaz, ${ }^{*}$ Karla Gabriela Peniche Moguel, ${ }^{*}$ Gerardo Rivera Solís, ${ }^{*}$ Eduardo Alberto González Escudero, ${ }^{*}$ Enrique Antonio Martínez Rodríguez, ${ }^{\ddagger}$ Enrique Monares Zepeda, ${ }^{\ddagger}$ María Verónica Calyeca Sánchez*

\section{RESUMEN}

La pandemia por COVID-19 es un parteaguas en nuestra historia moderna; la catástrofe mundial que ha generado tiene fecha de inicio, pero aún no fecha final; las anécdotas, los aciertos, los errores, las enseñanzas son parte de nuestro hacer cotidiano y ahora, por la situación apremiante de la pandemia, se han ido intensificando al tener repercusión en el ámbito laboral e, incluso, personal. A través de esta investigación con técnica de encuesta se pretende exponer la problemática en el ámbito emocional que aqueja a los trabajadores de la salud en tiempos de COVID-19.

Palabras clave: Pandemia, catástrofe, investigación, salud, COVID-19.

\section{ABSTRACT}

The COVID-19 pandemic is a watershed in our modern history, the global catastrophe it has generated has a start date but not yet an end date; anecdotes, successes, mistakes, and teachings are part of our daily work and that now due to the pressing situation of the pandemic have been intensifying, having repercussions in the workplace and even personal. Through this investigation with a survey technique, the aim is to expose the emotional problems that afflict health workers in the time of COVID-19.

Keywords: Pandemic, catastrophe, research, health, COVID-19.

\section{RESUMO}

A pandemia de COVID-19 é um divisor de águas em nossa história moderna; a catástrofe global que gerou tem uma data de início, mas ainda não uma data de término; anedotas, sucessos, erros e ensinamentos fazem parte de nossas atividades diárias e, agora, devido à situação premente da pandemia, estão se intensificando, repercutindo no local de trabalho e até na esfera pessoal. Por meio dessa investigação, com uma técnica de pesquisa, o objetivo é expor os problemas emocionais que atingem os profissionais de saúde na época do COVID-19.

Palavras-chave: Pandemia, catástrofe, pesquisa, saúde, COVID-19.

\section{INTRODUCCIÓN}

Todos los días tengo un tema en común con mis compañeros del hospital: COVID-19 (acrónimo del inglés coronavirus disease 2019). Nos sentimos presionados por esta experiencia única para la mayoría de nosotros, estresados y con sentimientos asociados (miedo, ansiedad, enojo, frustración), pero de ninguna manera ello es un reflejo de debilidad o de incompetencia para realizar nuestro trabajo; la salud mental es tan importante como la física para mantenernos en equilibrio. De hecho, un estudio que incluyó 34 hospitales chinos reportó que el personal de salud (médicos y enfermeras) sufría angustia $(71.5 \%)$, depresión (50.4\%), ansiedad (44.6\%) e insomnio (34\%). En esta encuesta realizada en traba-

\footnotetext{
* Instituto Mexicano del Seguro Social (IMSS), Unidad Médica de Alta Especialidad (UMAE), Hospital de Especialidades Núm. 14, Centro Médico Nacional «Adolfo Ruiz Cortines", Veracruz. Veracruz, México.

¥ Centro Médico ABC. Ciudad de México, México.

Recepción: 05/05/2020. Aceptación: 22/05/2020.

www.medigraphic.com/medicinacritica
}

jadores de la salud podemos notar la carga psicológica del personal comprometido en la atención de pacientes con COVID-19. ${ }^{1}$

La preocupación en el personal de salud es similar: miedo a contagiarse y miedo al contagio de familiares, amigos o compañeros. Lo anterior conlleva renuencia al trabajo, dejando de lado el interés económico y pensando en la renuncia laboral como medida desesperada en esta pandemia. La falta de profesionalismo o vocación no son las razones, sino que sencillamente faltan equipos de protección personal (EPP), insumos, medicamentos específicos, equipos para ventilación mecánica e, incluso, la ausencia de un espacio físico «áreas específicas» disponibles para la atención de pacientes COVID-19.2-4 El gran número de casos sospechosos y confirmados y la carga abrumadora de trabajo, por supuesto que también influyen, aunque son situaciones del día a día en nuestros hospitales públicos, incluso antes de la pandemia de COVID-19; aun así, se tiene calidad y calidez en la atención del paciente, siempre dignas de reconocérsele al personal de salud.

Ya lo dijo Paracelso: «todo es veneno y nada es veneno, sólo la dosis hace el veneno " y la sobrecarga de información puede ser "venenosa», porque genera estrés; me refiero a la «infobesidad» de las diferentes redes sociales, la cual ocasiona ansiedad y angustia en mucho personal de salud. Sitios oficiales, fuentes confiables, no escuchar o transmitir rumores pueden ayudar a minimizar el miedo —lo sabemos bien-, pero aislarnos de mala información en la actualidad resulta imposible. ${ }^{5}$

\section{MATERIAL Y MÉTODOS}

Realizamos una encuesta personal, vía correo electrónico y redes sociales, la cual incluyó 10 preguntas que fueron hechas basándonos en las «dudas» del día a día de diferentes hospitales donde laboramos o donde laboran compañeros y amigos. En el personal entrevistado, el requisito indispensable fue que atendieran pacientes sospechosos o confirmados COVID-19. Los encuestados fueron médicos y enfermeras; los servicios en los que laboran son: urgencias, piso (hospitalización), UCl (Unidad de Cuidados Intensivos) y quirófano. Incluimos hospitales públicos y privados. Las preguntas y respuestas de la encuesta están en el Anexo 1. 


\section{RESULTADOS}

Se encuestaron a 150 elementos del personal de salud (Tabla 1); de ellos, $66.6 \%$ considera que el origen del coronavirus 2 del síndrome respiratorio agudo grave (SARS-CoV-2) es una zoonosis, aunque $26.6 \%$ considera que es una guerra entre naciones poderosas y 4 y $2.66 \%$ refiere que es un castigo de Dios o que no existe, respectivamente.

La mayoría (63.3\%) del personal de salud entrevistado piensa que sólo $25 \%$ del personal de sus hospitales se encuentra capacitado para atender pacientes con COVID-19, $24.6 \%$ opina que $50 \%, 8.66 \%$ considera que $75 \%$ y sólo $3.33 \%$ piensa que $100 \%$.

La ansiedad fue el sentimiento más frecuente en $65.33 \%$, pero $17.33 \%$ presentó enojo, $9.33 \%$ pánico y a $8 \%$ le fue indiferente. La principal carencia según la percepción del personal es el equipo de protección personal (EPP) (54.66\%), seguida del equipo tecnológico o ventiladores (26.66\%); $12 \%$ percibió que faltaba recurso humano (médicos, enfermeras, inhaloterapeutas) y $6.66 \%$ medicamentos.

La principal preocupación personal de médicos y enfermeras es infectar a algún miembro de su familia con $68 \%$, infectarse $18 \%$, morir $12 \%$ y sólo $2 \%$ está preocupado por su economía en estos momentos. Además $65.33 \%$ sabe que el nivel socioeconómico es indistinto como factor de riesgo para esta enfermedad, $19.33 \%$ considera que el nivel socioeconómico bajo es un factor de riesgo para contagiarse, 10.66 y $3.88 \%$ consideran al nivel socioeconómico medio y alto como factor de riesgo.

Tabla 1: Características demográficas y su distribución.

\begin{tabular}{lc}
\hline Variable & $\begin{array}{c}\text { Total }(\mathrm{N}=150) \\
\mathrm{n}(\%)\end{array}$ \\
\hline Género & \\
Hombre & $90(60.0)$ \\
Mujer & $60(40.0)$ \\
Grado académico & \\
Médico adscrito & $50(33.3)$ \\
Intensivista & 20 \\
Medicina de urgencias & 10 \\
Medicina interna & 10 \\
Anestesiología & 10 \\
Médico residente & $40(26.6)$ \\
Enfermera general & $20(13.3)$ \\
Enfermera especialista & $40(26.6)$ \\
Tipo de hospital & \\
Público & $119(79.3)$ \\
Privado & $31(20.6)$ \\
Área del Hospital CovID-19 & \\
Urgencias & $42(28.0)$ \\
Terapia intensiva & $73(48.6)$ \\
Hospitalización & $21(14.0)$ \\
Quirófano & $14(9.3)$ \\
Tipo de atención & \\
Presencial & $150(100.0)$ \\
A distancia & 0 \\
\hline
\end{tabular}

Entre los entrevistados la principal fuente de información fueron los textos médicos (64\%), televisión y radio $(20 \%)$, redes sociales $(14.66 \%)$ y $1.33 \%$ utilizó como fuente de información a sus compañeros, amigos y familiares. Así también, $58.66 \%$ sabe que la edad para contagiarse es indistinta, el $22.66 \%$ considera que la enfermedad afecta al adulto maduro (36 a 59 años), $16 \%$ a los mayores de 60 años, $2.66 \%$ al adulto joven (18 a 35 años) y no hubo quien opinara que los menores de 18 años tienen menor riesgo de infectarse.

Un porcentaje de $84.66 \%$ de los encuestados está de acuerdo que el personal mayor de 60 años, así como con enfermedades cronicodegenerativas, inmunodeficiencias o alguna otra condición fuera a casa como medida de protección, pero $15.33 \%$ no está de acuerdo con esta medida.

Finalmente, $52 \%$ considera que el principal mecanismo de contagio es tocar ojos, nariz y boca con manos contaminadas, $37.77 \%$ las gotas de Flügge y aerosoles suspendidas en el aire por tiempo determinado; $1.33 \%$ piensa que es porque el virus se encuentra suspendido permanentemente en el aire y otro $1.33 \%$ cree que se puede contagiar con tan sólo tocar al paciente.

\section{DISCUSIÓN}

Nos preguntamos: ¿qué sigue después de la pandemia de COVID-19? Es importante mencionar que después de haber estado con ventilación mecánica invasiva (VMI) en la UCI por un problema pulmonar, 6 de cada 10 pacientes presentará trastorno de estrés postraumático (TEPT) de importancia clínica, y además que la mitad de estos pacientes recibirá apoyo psiquiátrico y farmacológico en los dos años posteriores al alta hospitalaria.

EI TEPT no es exclusivo de personas que van a la guerra o de personas que son atacadas sexualmente, de hecho, es tan común o todavía más común en los pacientes que "sobreviven a la UCl» que en los soldados, pero es algo que la mayoría de los médicos aún no apreciamos con facilidad. Nunca debemos olvidar a los familiares, pues ellos «sufren» la enfermedad del paciente con la misma intensidad, pero en diferente escenario. De esta manera, $70 \%$ de los familiares presenta ansiedad, 35\% depresión y 35\% TEPT.

Los que nos dedicamos a la atención de pacientes críticamente enfermos (en cualquier parte del hospital) sabemos que hoy la sobrevida es mayor, pero va de la mano con un incremento de secuelas psíquicas, las cuales impactan en la calidad de vida después de la enfermedad crítica. 6,7 Los trabajadores de la salud no estamos exentos de presentar efectos adversos fisiológicos y psicológicos del estrés ambiental que se vive en tiempos de COVID-19. Lo anterior incrementa el riesgo de sufrir problemas neurológicos, psiquiátricos y cardiovasculares. ${ }^{8}$ 
La mejor manera de entender qué es lo que más preocupa a los profesionales de la salud es preguntar. Durante la primera semana de la pandemia de COVID-19 en Estados Unidos, en una encuesta realizada, se documentaron ocho causas de ansiedad. Éstas fueron: 1) acceso al EPP apropiado, 2) estar expuesto al COVID-19 en el trabajo y llevar la infección a su familia, 3) no tener acceso rápido a las pruebas si desarrollan síntomas y temor concomitante de propagar la infección en el trabajo, 4) incertidumbre si su organización respaldará o atenderá sus necesidades personales y familiares si desarrollan infección, 5) acceso a cuidado infantil durante el aumento de las horas de trabajo y el cierre de escuelas, 6) apoyo para otras necesidades personales y familiares a medida que aumentan las horas de trabajo y las demandas (alimentación, hidratación, alojamiento, transporte), 7) poder proporcionar atención médica competente si se despliega en una nueva área (por ejemplo, enfermeras sin experiencia en atención de pacientes críticamente enfermos que tengan que realizar esta función) y 8) falta de acceso a información y comunicación actualizadas. ${ }^{9}$ Incluso poblaciones con otra idiosincrasia, como la cultura europea, ha lidiado con esta problemática emocional y existencial de trastornos de ansiedad, así como con trastornos del sueño y trastornos conductuales que merman la calidez y calidad humana. ${ }^{10}$ Como podemos observar las inquietudes del personal de salud en otros países no distan de las del personal de salud de nuestro México. Reconocer las necesidades del personal operativo permitirá a las diferentes áreas administrativas de los hospitales desarrollar enfoques específicos para abordar estas inquietudes y brindar apoyo a su fuerza laboral de atención médica. ${ }^{11,12}$

Entonces, ¿qué sigue después de la pandemia de COVID-19? La respuesta sería: otra pandemia de problemas emocionales y conductuales en todas las personas que de una u otra manera estuvimos involucrados; por ello, los sistemas de salud público y privado deberán desarrollar estrategias para atender esta subsecuente pandemia, considerando que el impacto sobre la salud mental puede ocurrir inmediatamente después y persistir por un largo periodo de tiempo. ${ }^{9}$ Por último, «la salud es un estado de completo bienestar físico, mental y social, y no solamente la ausencia de afecciones o enfermedades»; la salud mental es el parteaguas para una existencia de calidad, adicionada con actitud y resiliencia que nos permita sobrellevar la situación actual y encontrar una nueva perspectiva para continuar.

\section{CONCLUSIÓN}

La pandemia de COVID-19 es una crisis de salud que dejará estigmas emocionales, no sólo en la sociedad, sino también en el personal de salud. La cuarentena y lo que ésta conlleva ha cambiado abruptamente la vida diaria de las personas, pero los hospitales colapsados y la gran cantidad de muertes que vemos diariamente están marcando la vida y las emociones de médicos y enfermeras. Los sentimientos, buenos o malos, no se pueden evitar, pero sí los podemos controlar, por lo que todas las emociones que percibimos en esta pandemia son parte de ella y al final quedarán atrás. Lo importante es evitar el comportamiento egoísta entre nosotros, entre el personal de salud y con nuestra sociedad. En tiempos de amenaza que no domine el impulso para ayudarse a uno mismo a toda costa, sino el impulso para ayudar a los demás. Recuerda: te cuidas, me cuidas... me cuido, te cuido... juntos atendemos a nuestros pacientes.

\section{BIBLIOGRAFÍA}

1. Lai J, Ma S, Wang Y, Cai Z, Hu J, Wei N. Factors associated with mental health outcomes among health care workers exposed to coronavirus disease 2019. JAMA Netw Open. 2020;3(3):e203976.

2. Maunder R, Hunter J, Vincent $L$, et al. The immediate psychological and occupational impact of the 2003 SARS outbreak in a teaching hospital. CMAJ. 2003;168(10):1245-1251.

3. Bai Y, Lin CC, Lin CY, Chen JY, Chue CM, Chou P. Survey of stress reactions among health care workers involved with the SARS outbreak. Psychiatr Serv. 2004;55(9):1055-1057. doi: 10.1176/appi.ps.55.9.1055.

4. Lee AM, Wong J, McAlonan GM, Cheung V, Cheung C, Sham PC, et al. Stress and psychological distress among SARS survivors 1 year after the outbreak. Can J Psychiatry. 2007;52(4):233-240. doi:org/10.1177/070674370705200405

5. Organización Panamericana de la Salud (OPS); Organización Mundial de la Salud (OMS) [sitio web]. Consideraciones psicosociales y de salud mental durante el brote de COVID-19. 2020 [actualizado 12 de marzo del 2020].

6. Bienvenu OJ, Gellar J, Althouse BM, Colantuoni E, Sricharoenchai T, Mendez-Tellez PA, et al. Post-traumatic stress disorder symptoms after acute lung injury: a 2-year prospective longitudinal study. Psychol Med. 2013;43(12):2657-2671.

7. Torres J, Carvalho D, Molinos E, Vales C, Ferreira A, Dias $\mathrm{CC}$, et al. The impact of the patient post-intensive care syndrome components upon caregiver burden. Med Intensiva. 2017;41(8):454-460.

8. Oken BS, Chamine I, Wakeland W. A systems approach to stress, stressors and resilience in humans. Behav Brain Res. 2015;282:144-154.

9. Shanafelt T, Ripp J, Trockel M. Understanding and addressing sources of anxiety among health care professionals during the COVID-19 pandemic. JAMA. 2020;323(21):2133-2134. doi:10.1001/jama.2020.5893

10. Casagrande M, Favieri F, Tambelli R, Forte G. The enemy who sealed the world: Effects quarantine due to the COVID-19 on sleep quality, anxiety, and psychological distress in the Italian population. Sleep Med. 2020. doi: 10.1016/j.sleep.2020.05.011.

11. Huang Y, Zhao N. Generalized anxiety disorder, depressive symptoms and sleep quality during COVID-19 outbreak in china: a web-based cross-sectional survey. Psychiatry Res. 2020;288:112954.

12. Adams JG, Walls RM. Supporting the health care workforce during the COVID-19 global epidemic. JAMA. 2020;323(15):1439-1440.

\author{
Correspondencia: \\ Dr. Jesús Salvador Sánchez Díaz \\ Av. Cuauhtémoc $\mathrm{S} / \mathrm{N}$, \\ Col. Formando Hogar, 91810, Veracruz, Ver. \\ Tel: 2299342031 \\ E-mail: drsalvadorsanchezdiaz@gmail.com
}




\section{Anexo 1.}

1. ¿Cuál considera usted que sea el origen de la enfermedad COVID-19?
a. Castigo de Dios
b. Zoonosis
c. Guerra entre naciones poderosas
d. No existe

2. ¿Qué porcentaje del personal médico y de enfermería de su hospital considera usted está capacitado para la atención de pacientes con COVID-19?
a. $25 \%$
b. $50 \%$
c. $75 \%$
d. $100 \%$

3. ¿Qué sentimiento le genera la pandemia por COVID-19?
a. Enojo
b. Ansiedad
c. Pánico
d. Me es indiferente

4. ¿Cuál considera usted que es la principal carencia de su hospital?
a. Equipo tecnológico (ventiladores)
b. Equipo de protección personal (EPP)
c. Recurso humano (médico, enfermeras, inhalote- rapeutas)
d. Medicamentos e insumos

5. ¿Cuál es su principal preocupación personal en esta pandemia de COVID-19?
a. Infectarme
b. Infectar a la familia
c. Morirme
d. Mi economía

6. ¿Cuál considera usted que sea el nivel socioeconómico que tiene más riesgo de infección por COVID-19?
a. Nivel económico alto
b. Nivel económico medio
c. Nivel económico bajo
d. Es indistinto

7. ¿Cuál es su principal fuente de información respecto a la pandemia del COVID-19?
a. Redes sociales
b. Televisión y radio
c. Compañeros/amigos/familiares
d. Textos médicos

8. ¿Qué población considera usted tiene mayor riesgo de infectarse por COVID-19?
a. Menores de 18 años
b. Adulto joven (18 a 35 años)
c. Adulto maduro (36 a 59 años o más)
d. Mayores de 60 años
e. La edad para contagio es indistinta

9. ¿Está usted de acuerdo que el personal de salud mayor de 60 años con enfermedades crónico-degenerativas, inmunodeficiencias o alguna otra condición haya ido a casa como medida de protección?
a. Sí
b. No

10. ¿Cuál considera usted que es el principal mecanismo de contagio por coronavirus?

a. El virus que se encuentra suspendido permanentemente en el aire

b. El virus que se encuentra en gotas de Flügge y aerosoles por un tiempo determinado en el aire

c. Con sólo tocar al paciente

d. Tocar mis ojos, nariz o boca con manos contaminadas 